\title{
EchoGéo
}

28 | 2014

Police : les espaces de l'ordre, l'ordre en espace

\section{Judiciarisation des personnes itinérantes à Québec : une géographie des pratiques policières répressives au service de la revitalisation}

\section{Catherine Chesnay, Céline Bellot et Marie-Ève Sylvestre}

\section{CpenEdition}

\section{Journals}

Édition électronique

URL : https://journals.openedition.org/echogeo/13826

DOI : $10.4000 /$ echogeo. 13826

ISSN : 1963-1197

Éditeur

Pôle de recherche pour l'organisation et la diffusion de l'information géographique (CNRS UMR 8586)

Référence électronique

Catherine Chesnay, Céline Bellot et Marie-Ėve Sylvestre, « Judiciarisation des personnes itinérantes à Québec : une géographie des pratiques policières répressives au service de la revitalisation », EchoGéo [En ligne], 28 | 2014, mis en ligne le 08 juillet 2014, consulté le 31 juillet 2021. URL : http:// journals.openedition.org/echogeo/13826 ; DOI : https://doi.org/10.4000/echogeo.13826

Ce document a été généré automatiquement le 31 juillet 2021.

EchoGéo est mis à disposition selon les termes de la licence Creative Commons Attribution - Pas d'Utilisation Commerciale - Pas de Modification 4.0 International (CC BY-NC-ND) 


\title{
Judiciarisation des personnes itinérantes à Québec : une géographie des pratiques policières répressives au service de la revitalisation
}

\author{
Catherine Chesnay, Céline Bellot et Marie-Ève Sylvestre
}

\section{NOTE DE L'AUTEUR}

Les auteures tiennent à remercier chaleureusement les évaluateurs anonymes qui par leurs commentaires leur ont permis d'asseoir leurs réflexions sur un territoire qui n'est pas le leur : celui de la géographie.

\section{Introduction}

1 La pauvreté fait peur et nuit aux affaires... C'est dans ce cadre que les villes cherchent depuis quelques décennies à mettre en place des politiques et des pratiques à l'endroit des populations itinérantes ${ }^{1}$ en vue de les sortir des rues. Or, cette sortie de rue tient bien moins à la mise en œuvre d'actions publiques protectrices, solidaires et inclusives, qu'à la mise en œuvre d'actions publiques répressives, excluantes et ciblées. Dès lors, comprendre la judiciarisation des personnes itinérantes constitue un enjeu majeur dans le développement des connaissances sur la manière dont s'exerce le contrôle de l'espace public dans nos sociétés contemporaines.

2 En effet, la montée des questions de sécurité, de la gestion pénale des problèmes sociaux et de la revitalisation urbaine ont largement contribué au développement de nouvelles politiques et pratiques à l'égard de l'occupation de l'espace public (Harcourt, 
2006 ; Bellot et al., 2005). Que ce soit par les transformations des pratiques policières, notamment en regard de la police de proximité, par le développement de la sécurité privée dans des espaces publics ou quasi publics, par la mise en place des stratégies de vidéosurveillance et autres types de contrôles technologiques, par la création des règlements municipaux ou par des lois provinciales et par les politiques urbaines de revitalisation, il est possible de constater une reconfiguration du contrôle social en particulier à l'égard des personnes qui utilisent l'espace public comme espace de vie ou de travail comme le font les personnes itinérantes (Parazelli et al., 2013; Harcourt, 2006 ; Amster, 2004).

3 Dans cette perspective, la production de la sécurité est devenue pour les villes un enjeu majeur tant pour soutenir leur attractivité, que pour assurer une protection de leurs citoyens à l'égard d'une pauvreté menaçante (Smith, 1996). L'objectif de cet article en démontrant comment les pratiques de judiciarisation de l'itinérance à Québec se superposent aux dynamiques de revitalisation urbaine mises en œuvre dans cette ville, permet de s'interroger sur la production d'un ordre social urbain imposé plutôt que négocié, qui tend à exclure et discriminer les populations les plus pauvres plutôt que les inclure.

\section{L'ancrage sécuritaire de la revitalisation des villes}

Les politiques de revitalisation, menées de par le monde, visent le plus souvent les mêmes objectifs : restaurer un ordre social perdu, en cherchant à réduire les effets de la pauvreté urbaine, et accroître la qualité de vie dans les milieux urbains (Bacqué et al., 2003). Ainsi, à travers de multiples interventions intersectorielles (urbanistiques, architecturales, économiques, sociales, etc.), la revitalisation vise à soutenir la transformation physique, fonctionnelle et symbolique d'un quartier dans une perspective de pacification urbaine notamment par la promotion d'une mixité sociale (Divay et Séguin, 2004). En effet, si la pauvreté apparaît comme un des vecteurs de la dégradation physique et symbolique d'un quartier, l'arrivée de nouvelles populations de la classe moyenne, de nouvelles infrastructures économiques, devient dans la revitalisation, le moteur de la relance du quartier.

Cette nécessité de la revitalisation des quartiers apparaît d'autant plus impérieuse que dans un contexte de compétitivité internationale, les grands centres urbains déploient une stratégie de spécialisation économique dans le but d'attirer des investissements étrangers (Rousseau, 2008; Atkinson, 2003 ; Sassen, 2002 ; Smith, 1996). Pour ce faire, les politiques urbaines ne doivent pas seulement se concentrer sur l'attraction des entreprises, mais aussi sur le développement d'une qualité de vie, d'environnement urbain, de vie culturelle, etc. perçus comme nécessaire à l'attraction et à la rétention d'une élite pour ces entreprises (Rousseau, 2008 ; Bacqué et al., 2003 ; Sassen, 2002).

6 La question des politiques urbaines et de la sécurisation des espaces publics devient alors un enjeu majeur à la fois pour attirer mais aussi accueillir ces nouveaux commerçants et résidents (Parazelli, 2010 ; Doherty et al., 2008 ; Wacquant, 2004 ; Mary, 2003 ; Smith, 1996). En effet, dans ce marché mondial des grandes villes, la rue, l'espace public devient une sorte d'image de la ville qu'il importe de contrôler et bien sûr d'embellir. L'évacuation ou l'expulsion de populations marginalisées (travailleurs du sexe, toxicomanes, jeunes de la rue) est vue alors comme nécessaire en terme de 
développement urbain. La visibilité des personnes itinérantes constitue un obstacle à cette image valorisante (Doherty et al., 2008).

7 Par conséquent, le contrôle des espaces publics (rues, parcs, places) à travers des dispositifs policiers mais aussi urbanistiques et juridiques est devenu un outil de cette revitalisation au même titre que le travail d'embellissement des quartiers. Québec n'échappe à cette réalité, d'autant plus que le travail de revitalisation de la ville cherche à mettre l'accent sur le tourisme international, en valorisant l'image d'une ville entre l'Europe et l'Amérique, première ville d'Amérique du Nord (Freedman, 2009 ; Ville de Québec, 2005).

Or, les effets de ces politiques de revitalisation sur les populations originelles, pauvres et marginalisées de ces quartiers sont souvent ignorés (Freedman, 2009). Certes, le terme "cité revancharde" (revanchist city) est couramment utilisé dans les écrits américains pour désigner cette appropriation agressive et vengeresse des centres urbains par les classes moyennes supérieures au détriment des couches les plus marginalisées de la société (DeVertreuil et al., 2008 ; Smith, 1996). Mais, au Canada et au Québec, peu d'études se sont intéressées à cette question. Freedman (2009) dans son étude menée entre 2001 et 2006 sur l'effet de la revitalisation d'un quartier à Québec sur les personnes itinérantes conclut que l'opération de revitalisation a permis aux personnes itinérantes de retrouver une fierté dans leur quartier et en eux-mêmes mais qu'elle a aussi conduit à les repousser en dehors du quartier.

9 Ainsi, les logiques de sécurisation qu'introduisent, dans certains quartiers, les politiques de revitalisation, en mettant l'accent sur le contrôle de l'espace public paraissent cibler les populations marginalisées, notamment les personnes itinérantes, ce qui n'est pas sans conséquences pour ces dernières.

\section{Le contrôle de l'espace public : un enjeu pour les personnes itinérantes}

10 L'histoire de l'oscillation entre l'aide et le contrôle de l'itinérance commence avec le Moyen Âge, puisque, dès cette époque, le lien entre errance ou pauvreté et criminalité constitue une manière de définir "la mauvaise pauvreté » et donc d'en assurer un traitement pénal (Gueslin, 2013; Fecteau, 2004; Wagniart, 1999). C'est autour de ce travail de construction de la différenciation sociale entre les «bons» et les «mauvais pauvres » que se bâtit la légitimation de l'usage de la répression (Wagniart, 1999; Castel, 1995). Le domicile et le travail sont alors les clés qui distinguent les individus. Cependant, ce contrôle de l'itinérance connaît des transformations majeures, notamment dans le contexte de la gestion des espaces publics dans nos villes contemporaines et dans leurs quartiers revitalisés (Laberge et Roy, 2001).

\section{La construction de l'itinérance comme un problème d'ordre public}

11 Ce cadre historique demeure d'actualité car l'itinérant apparaît comme une menace pour l'ordre établi dans la mesure où sa présence dans l'espace public vient renforcer le sentiment d'insécurité des autres citoyens (Gueslin, 2013 ; Amster, 2004 ; Fecteau, 2004). En effet, la vie urbaine moderne s'est construite autour de l'idée que l'espace public devait être celui de la mobilité, de la circulation en devenant des « purified social 
spaces» (Mitchell, 2003; Sibley, 1995), dans lesquels les personnes passent, pour rejoindre les deux espaces de sécurité que sont le domicile et le travail. L'utilisation, par les personnes itinérantes, de l'espace public comme un espace de vie renforce alors l'idée d'un désordre à un double titre. D'une part, le manque d'espaces privés des personnes itinérantes témoigne de leur déviance. D'autre part, leur présence dans l'espace public, en renforçant leur visibilité, renforce leur caractère dérangeant et menaçant pour l'ordre public (Mitchell, 2003). Cette représentation de l'itinérant comme une catégorie sociale à risque contribue alors à la légitimation d'une gestion pénale comme outil de défense de l'ordre social (Hacourt, 2006 ; Amster, 2003 ; Mitchell, 1997).

12 Certes, la question de la gestion policière et pénale des problèmes sociaux n'est pas récente (Bernard, 2005; Wacquant, 2004 ; Beckett et Western, 2001). De nombreux auteurs après Foucault, ont démontré comment le système pénal œuvrait à la mise en place de stratégies de contrôle des personnes et des populations dans une logique de normalisation et de disciplinarisation des situations de pauvreté (Wacquant, 2004 ; Chantraine, 2004 ; Vanneste, 2001). Pour autant, les configurations contemporaines de ce contrôle social s'ancrent dans des pratiques répressives ciblées tant au plan des populations que des quartiers concernés (Beckett et Western, 2001).

13 Construite autour des enjeux de l'insécurité et des incivilités, cette utilisation néolibérale du droit pénal témoigne des enjeux normatifs et institutionnels qui prévalent dans nos sociétés contemporaines pour réaliser l'apparente difficulté du «vivreensemble » dans nos sociétés (Beckett, et Western, 2001).

\section{Le déploiement de la réaction policière et pénale}

14 La logique de la réaction pénale s'inscrit dans un cadre de défense sociale où l'important est donc d'identifier et de mettre à l'écart de la collectivité les situations, les comportements, mais bien davantage encore les personnes, susceptibles de perturber l'ordre social (Walby et Lippert, 2012; Blomley, 2010; Amster, 2004; Coleman, 2004 ; Bauman, 2000). L'accent est alors mis sur le contrôle et la protection de la collectivité, en visant la mise à l'écart des communautés, des personnes définies comme des menaces (Beckett et Herbert, 2008 ; Bellot et al., 2005; Wacquant, 2004). Rétablir l'ordre dans les villes est devenu une impérieuse nécessité pour contrer le laissez aller, terreau fertile des illégalismes et de la délinquance (Harcourt, 2006 ; Bellot et Morselli, 2002). Dans cette perspective, la désorganisation sociale devient un cadre théorique, permettant à la fois de définir les personnes qui vivent les problèmes sociaux comme des menaces à l'ordre établi, et dès lors, de légitimer des interventions répressives. La théorie de la vitre brisée de Wilson et Kelling (1982) va dans ce sens, en affirmant qu'en laissant la place aux désordres sociaux dans un quartier, on alimente la criminalité (Harcourt, 2006). A ce titre, les différentes politiques et pratiques de tolérance zéro fondées sur cette perspective théorique sont venues alimenter le recours à la répression pour contrôler les populations «menaçantes » pour l'ordre social (Wacquant, 2004).

$15 \mathrm{Au}$ Canada et au Québec, l'accessibilité des personnes itinérantes à l'espace public est devenue de plus en plus restreinte (Walby et Lippert, 2012; O'Grady et al., 2011; Sylvestre, 2010 ; Berti et Sommers, 2010 ; Bellot, et al., 2005 ; Collins et Blomley, 2003). Elle s'inscrit dans une contraction de l'espace public urbain par l'intermédiaire, entre 
autres, de la privatisation de cet espace public, ainsi que de l'instauration de divers dispositifs de contrôle de l'espace urbain, comme la transformation de places publiques en $\operatorname{parcs}^{2}$ (Sylvestre, 2010), la transformation du mobilier urbain ${ }^{3}$ ou encore des changements dans la signalisation routière afin de contrôler les allées et venues dans certaines rues du centre-ville (Berti et Sommers, 2010 ; Parazelli, 2010 ; Sylvestre, 2010 ; Collins et Blomley 2003). Certains de ces dispositifs de contrôle découlent de changements législatifs et réglementaires, menant ainsi à une prise en charge pénale du problème posé par la présence de personnes itinérantes dans l'espace public (Bellot et al., 2005).

Or, si de manière générale, les études sur le contrôle pénal des populations itinérantes se sont multipliées dans les dernières années pour rendre compte du bannissement des populations itinérantes dans les centres-villes (Beckett et Herbert, 2008 ; Bellot et al., 2005; Mitchell, 1997), peu d'études ont directement lié le bannissement à des dynamiques socio-spatiales des pratiques policières mises en œuvre dans le cadre des stratégies de revitalisation, dynamiques qui contribuent à la judiciarisation des personnes itinérantes.

\section{Méthodologie}

17 Notre recherche a permis de documenter quantitativement la judiciarisation des personnes itinérantes à Québec (Bernier et al., 2012). Pour ce faire, à partir de la banque de la cour municipale une extraction des constats d'infraction émis auprès des populations itinérantes a été réalisée. Et c'est à partir de ces données que cette étude appréhende la pratique policière répressive ${ }^{4}$. Dans un premier temps, l'extraction a été effectuée en utilisant les adresses civiques d'organismes communautaires qui interviennent auprès des personnes itinérantes à Québec. Or, comme il est moins courant à Québec d'utiliser les adresses des ressources (en comparaison à d'autres villes dans lesquelles nous avons utilisé la même méthode), une seconde extraction a été effectuée sur l'ensemble des contraventions reçues par ces personnes, indépendamment de l'adresse fournie au moment de l'émission du constat.

Utilisée pour d'autres terrains de recherche (Bellot et al., 2005), l'utilisation d'adresses civiques d'organisme comme critère d'extraction de données permet de s'assurer que les constats ont bel et bien été émis à des personnes en situation d'itinérance. Néanmoins, cette méthode présente le désavantage de ne recenser que les constats ayant été remis à des personnes ayant utilisé les adresses d'organismes communautaires comme adresse lors de la remise du constat. Ainsi, les constats remis à des personnes en situation d'itinérance qui ne fréquentent pas ces organismes, qui donnent leur ancienne adresse personnelle ou alors qui sont en situation de logement précaire (qui sont hébergées temporairement par un proche) ne sont pas recensés. Nos données représentent donc la pointe de l'iceberg du phénomène de la judiciarisation de l'itinérance et non un portrait exact.

Nous avons extrait les constats d'infractions émis entre le $1^{\text {er }}$ janvier 2000 et le 31 décembre 2010. Cette période permet de saisir l'évolution des pratiques au fil du temps et s'inscrit historiquement dans le cadre d'une stratégie de revitalisation du Quartier St Roch qui durant cette décennie a profondément transformé l'espace public, fréquenté par les personnes itinérantes. En effet, avant 1998, ce quartier disposait d'une large promenade commerciale, avec un toit, Mail Centre ville que les personnes 
itinérantes et marginalisées fréquentaient au quotidien. La suppression du toit, puis les opérations de revitalisation successives du quartier sont venues modifier la territorialité des personnes itinérantes (Freedman, 2009).

Dans notre banque de données originale ( $\mathrm{n}=3735$ constats pour 284 personnes), 1173 infractions (31,41\% de la banque) ont été exclues car le lieu d'infraction ${ }^{5}$ n'était pas inscrit dans le constat d'infraction. Nous avons aussi éliminé les constats pour lesquels le lieu était inexistant (par exemple intersection de deux rues parallèles, numéro qui n'existe pas) ou alors les cas où l'adresse était un lieu de résidence, c'est-àdire qu'il y avait un numéro d'appartement indiqué. Ont donc été retenus les différents constats d'infraction pour lesquels un lieu d'infraction était mentionné lorsque ce lieu correspondait à une adresse non résidentielle. Nous avons aussi laissé de côté les constats qui étaient dans des quartiers pour lesquels il y avait moins de 5 constats. Par conséquent, l'échantillon pour lequel nous avons identifié une localisation de l'infraction sur le constat est de 2562 infractions et 257 individus.

21 Afin de dresser une cartographie des positions de l'ensemble des infractions étudiées, chaque constat de l'échantillon a été codé en fonction de l'adresse ou de l'intersection où l'infraction a eu lieu, puis les constats ont été regroupés en fonction des quartiers, tels qu'établis lors de la division de Québec en 2009 : division en six arrondissements, eux-mêmes divisés en quartiers ${ }^{6}$.

\section{Les résultats}

L'analyse des données quantitatives mais aussi des politiques et des outils juridiques pour la ville de Québec a permis d'établir pour la période 2000-2010 le cadre, les outils et les politiques de la judiciarisation mais aussi de saisir la dynamique socio-spatiale de ces pratiques, notamment en regard des stratégies de revitalisation de certains quartiers de Québec.

\section{Les outils juridiques et les pratiques policières}

À Québec, la judiciarisation de l'itinérance repose principalement sur l'utilisation des réglementations municipales qui portent sur les incivilités et les comportements indésirables, ainsi que sur l'usage du Code de sécurité routière (Sylvestre, 2010 ; Bernier et al., 2011). Ces réglementations sont nombreuses et sont issues des différentes villes composant actuellement la ville de Québec. Ainsi, avec les fusions municipales, chaque arrondissement avait conservé sa propre réglementation, ce qui explique que les libellés d'infraction sont multiples dans notre banque de données. En 2009, la ville de Québec adopte un nouveau Règlement sur la paix et le bon ordre, qui couvre tous les comportements considérés comme des incivilités. Le Règlement sur la paix et le bon ordre interdit de nombreux actes considérés comme des incivilités tels qu'avoir consommé ou être en train de consommer des drogues ou de l'alcool, flâner, vagabonder, dormir sur un banc de parc, se battre, faire du désordre, insulter ou injurier un agent de la paix, uriner, mendier, lancer un objet, jouer dans la rue ${ }^{7}$, porter une arme, etc. Les peines se situent entre $100 \$$ (80 euros environ) et $1000 \$$ (800 euros environ) et, en cas de récidive, la peine minimale est de $300 \$$ (240 euros environ).

Les pratiques de judiciarisation des personnes itinérantes sont marquées par différentes vagues d'opérations policières, qui remontent à la fin des années 1980 
(Bernier et al., 2011). En raison d'un réaménagement du centre-ville de Québec, la Place d'Youville était le principal lieu de rencontre des marginaux, particulièrement des punks et des squeegees ${ }^{8}$ à la fin des années 1980 et au début des années 1990 (Dufour, 1998). Considérés comme des personnages inquiétants et potentiellement dangereux, les squeegees et les punks avaient la réputation de faire fuir les touristes et de déranger les passants, nuisant ainsi au commerce et au tourisme dans le centre-ville de Québec (Ligue des droits et libertés, 2012; Bouchard, 1995). Ces jeunes, qui sont qualifiés au Québec de jeunes de la rue, occupent en groupe l'espace public et l'utilisent comme espace de vie, de travail et de socialisation (Parazelli, 2010). On les distingue des personnes itinérantes, considérées le plus souvent comme plus âgées et le plus souvent isolées. Par contre, ils sont une manifestation de l'itinérance, prise en compte notamment dans le cadre d'interventions portées par des organismes qui leur sont dédiés.

Dans l'objectif de "sécuriser » les lieux et de rassurer les citoyens, la ville de Québec et le service de police ont eu recours à des opérations répressives, largement médiatisées au fil des années (Bouchard, 1995). En 2006, le projet Respect a été développé par le Service de police de la ville de Québec. Il se veut ouvertement une lutte face aux incivilités, afin notamment d'éradiquer la pratique du squeegee. Avec la mise en place de cette politique, les contraventions remises sont systématiquement comptabilisées: aussi lorsqu'un jeune squeegee avait accumulé plus de 6 infractions à un règlement municipal, une accusation criminelle pour avoir troublé la paix pouvait être déposée (Bernier et al., 2011; Fleury, 2002). Cette pratique n'a pas été analysée dans notre recherche, mais elle doit néanmoins être prise en considération en raison de la judiciarisation élevée des jeunes qui pratiquent le squeegee à Québec (Bernier et al., 2011).

Les résultats de notre recherche (2000-2010) montrent une augmentation de $300 \%$ des constats émis à l'encontre des personnes itinérantes à Québec, avec un pic en 2006, année de l'adoption du projet Respect (Bernier et al., 2011). Ainsi, si au début de la période, les contraventions émises étaient au nombre de 71, elles sont en 2010 au nombre de 348 comme le montre l'illustration 1.

Illustration 1 - Nombre de constats d'infraction par année

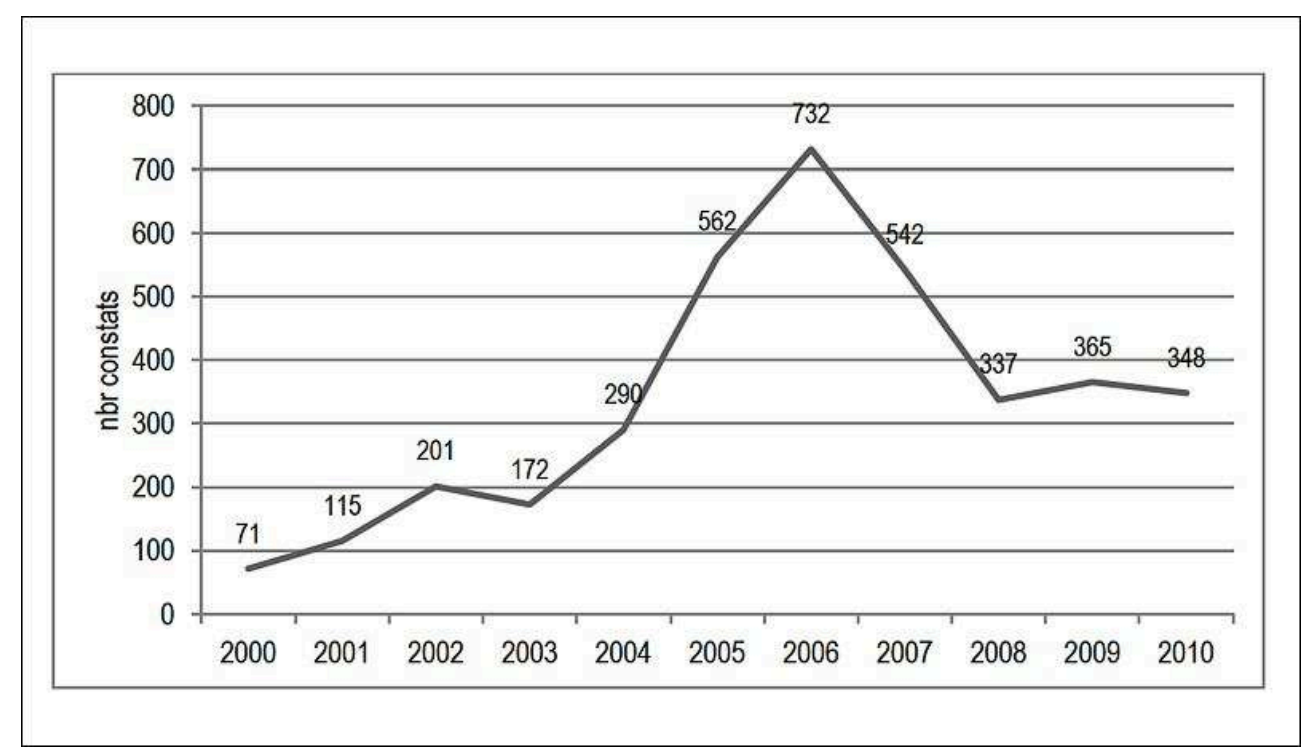


Sur le total des constats d'infraction, la majorité des constats (environ $95 \%$ ) sont émis en fonction de la réglementation municipale, plus spécifiquement les règlements qui sont relatifs à la consommation d'alcool ( $37 \%$ des constats), de la mendicité ( $23 \%$, soit 827 constats), et des problèmes liés aux troubles de l'ordre public (10\%, soit 385) comme le montre le tableau 1. En outre, ce tableau permet de constater que ce sont les personnes de moins de 30 ans qui font davantage l'objet de judiciarisation à Québec.

Tableau 1 - Âge au moment de l'infraction à un règlement municipal (tous les constats)

\begin{tabular}{|c|c|c|c|c|c|c|c|}
\hline & $\begin{array}{l}\text { Moins de } 20 \\
\text { ans }\end{array}$ & $\begin{array}{lll}20 & \text { à } & 24 \\
\text { ans } & \end{array}$ & $\begin{array}{lll}25 & \text { à } & 29 \\
\text { ans } & \end{array}$ & $\begin{array}{lll}30 & \text { à } & 39 \\
\text { ans } & \end{array}$ & 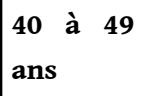 & $\begin{array}{l}50 \text { ans } \\
\text { et + }\end{array}$ & Total \\
\hline $\begin{array}{l}\text { Être en état d'ivresse } \\
\text { / l'alcool }\end{array}$ & $\begin{array}{l}80 \\
(19 \%)\end{array}$ & $\begin{array}{l}253 \\
(24 \%)\end{array}$ & $\begin{array}{l}226 \\
(32 \%)\end{array}$ & $\begin{array}{l}119 \\
(34 \%)\end{array}$ & $\begin{array}{l}235 \\
(58 \%)\end{array}$ & $\begin{array}{l}396 \\
(68 \%)\end{array}$ & $\begin{array}{l}1309 \\
(37 \%)\end{array}$ \\
\hline $\begin{array}{l}\text { Avoir flâné, } \\
\text { vagabondé }\end{array}$ & $\begin{array}{l}51 \\
(12 \%)\end{array}$ & $\begin{array}{l}103 \\
(10 \%)\end{array}$ & $\begin{array}{l}104 \\
(15 \%)\end{array}$ & $\begin{array}{l}33 \\
(9 \%)\end{array}$ & $\begin{array}{l}18 \\
(4 \%)\end{array}$ & $\begin{array}{l}19 \\
(3 \%)\end{array}$ & $\begin{array}{l}328 \\
(9 \%)\end{array}$ \\
\hline Troubler la paix & $\begin{array}{l}25 \\
(6 \%)\end{array}$ & $\begin{array}{l}61 \\
(6 \%)\end{array}$ & $\begin{array}{l}66 \\
(9 \%)\end{array}$ & $\begin{array}{l}58 \\
(17 \%)\end{array}$ & $\begin{array}{l}85 \\
(21 \%)\end{array}$ & $\begin{array}{l}90 \\
(16 \%)\end{array}$ & $\begin{array}{l}385 \\
(11 \%)\end{array}$ \\
\hline Mendier / solliciter & $\begin{array}{l}122 \\
(29 \%)\end{array}$ & $\begin{array}{l}397 \\
(38 \%)\end{array}$ & $\begin{array}{l}154 \\
(22 \%)\end{array}$ & $\begin{array}{l}99 \\
(28 \%)\end{array}$ & $\begin{array}{l}25 \\
(6 \%)\end{array}$ & $\begin{array}{l}30 \\
(5 \%)\end{array}$ & $\begin{array}{l}827 \\
(23 \%)\end{array}$ \\
\hline Squeegee & $\begin{array}{l}105 \\
(25 \%)\end{array}$ & $\begin{array}{l}141 \\
(13 \%)\end{array}$ & $\begin{array}{l}85 \\
(12 \%)\end{array}$ & $\begin{array}{l}5 \\
(1 \%)\end{array}$ & $\begin{array}{l}5 \\
(1 \%)\end{array}$ & $\begin{array}{l}0 \\
(0 \%)\end{array}$ & $\begin{array}{l}341 \\
(10 \%)\end{array}$ \\
\hline Autre infraction RM & $\begin{array}{l}33 \\
(8 \%)\end{array}$ & $\begin{array}{l}101 \\
(10 \%)\end{array}$ & $\begin{array}{l}79 \\
(11 \%)\end{array}$ & $\begin{array}{l}38 \\
(11 \%)\end{array}$ & $\begin{array}{l}40 \\
(10 \%)\end{array}$ & $\begin{array}{l}47 \\
(8 \%)\end{array}$ & $\begin{array}{l}338 \\
(10 \%)\end{array}$ \\
\hline TOTAL & $\begin{array}{l}416 \\
(100 \%)\end{array}$ & $\begin{array}{l}1056 \\
(100 \%)\end{array}$ & $\begin{array}{l}714 \\
(100 \%)\end{array}$ & $\begin{array}{l}352 \\
(100 \%)\end{array}$ & $\begin{array}{l}408 \\
(100 \%)\end{array}$ & $\begin{array}{l}582 \\
(100 \%)\end{array}$ & $\begin{array}{l}3528 \\
(100 \%)\end{array}$ \\
\hline
\end{tabular}

Nos données ont permis de vérifier une hypothèse courante, à savoir que la judiciarisation s'intensifie lors de la saison estivale, saison durant laquelle les personnes itinérantes sont plus visibles dans l'espace public - et durant laquelle l'espace public est plus sollicité pour des activités commerciales et culturelles (Bernier et al., 2011). L'été correspond aussi à l'accueil de nombreux touristes à Québec. Le déploiement d'une plus forte présence policière participe aussi au sentiment de sécurité pour les touristes. Notre recherche montre cependant qu'il ne s'agit pas simplement d'un accroissement de la surveillance policière mais bien d'un accroissement des pratiques répressives comme en témoigne l'illustration 2. 
Illustration 2 - Distribution par mois des constats d'infraction émis (2000-2010)

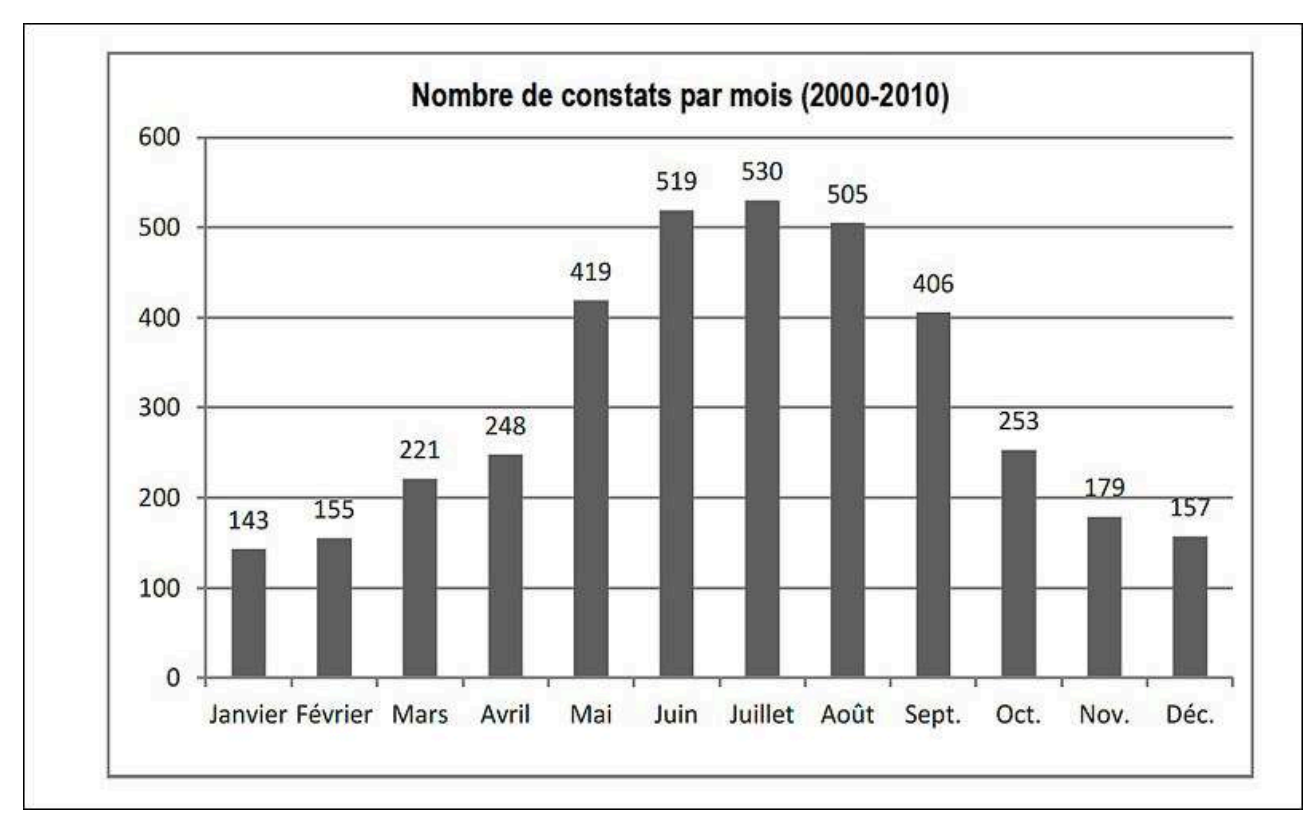

29 Les lignes qui suivent vont maintenant présenter dans quels quartiers, cette judiciarisation se réalise.

\section{La distribution géospatiale des constats d'infraction émis auprès des populations itinérantes}

L'analyse spatiale des constats émis, qui s'appuie sur l'étude de 2562 constats d'infraction, a permis d'établir que l'arrondissement de la Cité-Limoilou, soit un quartier central de Québec, est le principal arrondissement concerné par la judiciarisation de l'itinérance. Les constats émis représentent 72,91\% des constats de notre banque de données. Pour autant, l'analyse plus précise par quartier permet de constater que les quartiers concernés dans le centre-ville sont par ordre d'importance St-Roch, Vieux-Québec, St-Jean-Baptiste, St-Sauveur. L'illustration 3(reprise de l'étude de Freedman, 2009) permet de rendre compte des différents quartiers touchés par la judiciarisation. Une rue, la rue Honoré Mercier, constitue un autre lieu de forte judiciarisation de l'itinérance. Or, cette rue marque la frontière entre le Vieux Québec et St-Jean Baptiste, soit les deux quartiers où nous avons retrouvé une forte judiciarisation de l'itinérance. Le cas de cette artère est particulièrement intéressant, puisque $29 \%$ des constats qui y sont émis le sont à une intersection spécifique, l'intersection de la rue St-Jean et de la rue Honoré Mercier. Les constats émis à cette intersection représentent donc $3,28 \%$ du total des constats de notre banque de données. 
Illustration 3- Le quartier Saint-Roch à Québec et l'emplacement de l'ancien mail Centre-ville

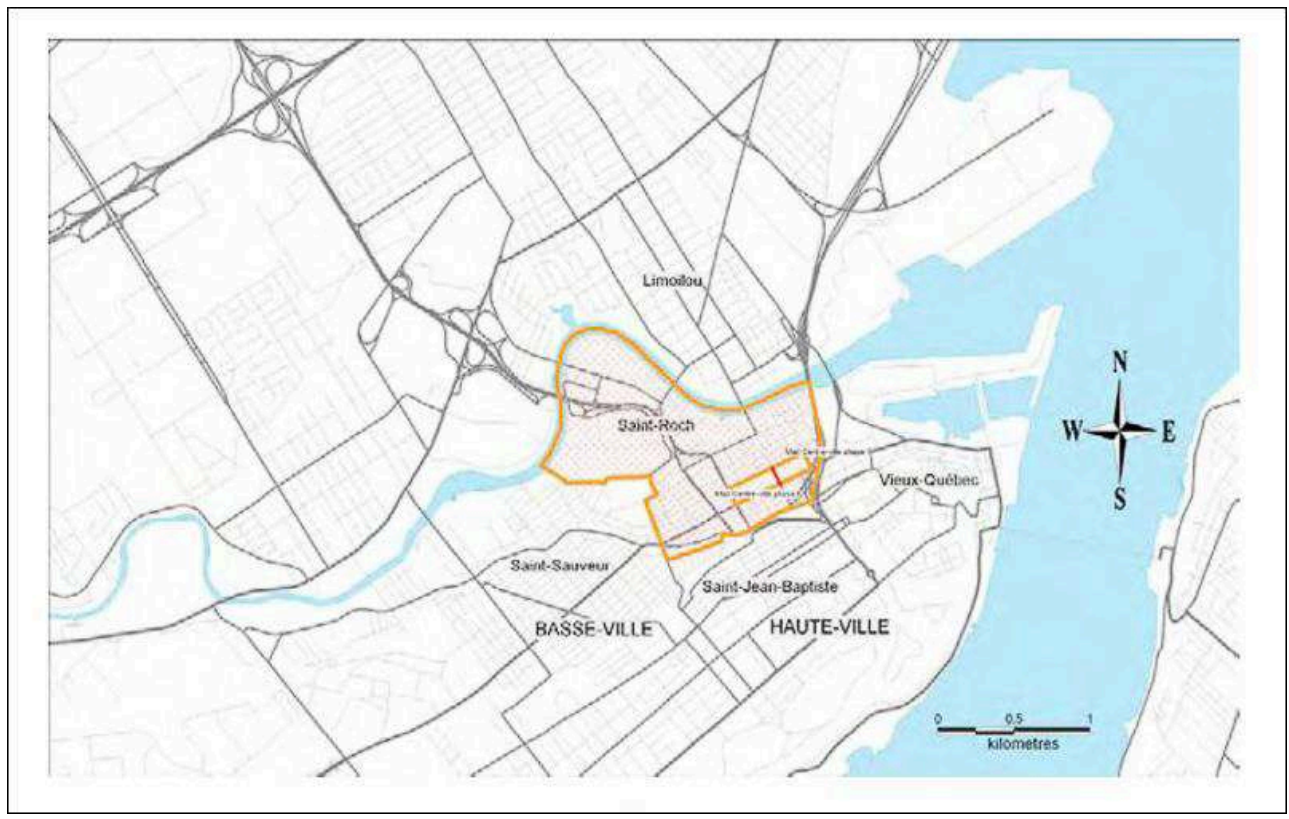

Sources : Freedman, 2009 ; Latig, Laboratoire du Département de géographie de l'Université Laval.

31 Le tableau 2 en dénombrant cette distribution géospatiale des constats d'infraction émis auprès des populations itinérantes rend compte du poids de chacun des quartiers dans cette judiciarisation.

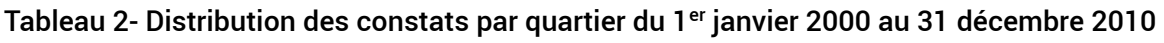

\begin{tabular}{|l|l|l|}
\hline Quartiers & Nombre & Pourcentage \\
\hline St-Roch & 994 & 38,80 \\
\hline Vieux-Québec (place d'Youville) & 586 & 22,87 \\
\hline St-Jean Baptiste & 221 & 8,63 \\
\hline St-Sauveur & 208 & 8,12 \\
\hline Rue Honoré Mercier & 288 & 11,24 \\
\hline Limoilou / & 116 & 4,53 \\
\hline Langelier & 90 & 3,51 \\
\hline Autres & 59 & 2,31 \\
\hline Total & 2562 & 100,00 \\
\hline
\end{tabular}

Cette distribution par quartier n'est pas surprenante dans la mesure où elle renvoie à la géographie du centre-ville touristique du Québec, à la fois dans les quartiers de ce qu'on nomme la Haute ville (Vieux Québec et St-Jean Baptiste) et dans ceux de la Basse 
Ville (St Roch et St Sauveur). Cependant, le fait que près de $39 \%$ des constats d'infraction sont émis dans le quartier St Roch montre comment des stratégies de revitalisation peuvent aussi être associées à des pratiques répressives à l'endroit des personnes itinérantes.

Cependant, c'est par l'analyse des infractions reprochées par quartier qu'il est possible de cerner davantage la géographie des pratiques policières répressives en s'intéressant à comprendre les comportements régulés.

\section{Distribution de la nature des infractions reprochées par quartier}

De manière globale, l'étude a permis de montrer que la judiciarisation de l'itinérance à Québec vise essentiellement les stratégies de survie des personnes (quête, squeegee) et la consommation d'alcool dans l'espace public (Bernier et al., 2011). En s'attardant aux trois zones géographiques (quartiers ou artères) où sont émis le plus de constats, une géographie des pratiques policières répressives se dessine, à travers la nature des comportements régulés, réifiant en ces zones une normativité du vivre-ensemble qui s'exprime moins dans les autres quartiers.

Tableau 3 - Distribution des infractions en fonction du type d'infraction en \%

\begin{tabular}{|c|c|c|c|c|c|c|c|}
\hline Quartier/Artères & $\begin{array}{l}\text { Ébriété } \\
\text { publique }\end{array}$ & $\begin{array}{l}\text { Flanâge/ } \\
\text { vagabondage }\end{array}$ & $\begin{array}{l}\text { Troubler la } \\
\text { paix }\end{array}$ & $\begin{array}{l}\text { Mendier/ } \\
\text { Solliciter }\end{array}$ & Squeegee & Autres & $\begin{array}{l}\text { Grand } \\
\text { Total }\end{array}$ \\
\hline $\begin{array}{l}\text { Intersection St- } \\
\text { Jean-Honoré } \\
\text { Mercier }\end{array}$ & 10,84 & 9,64 & 0,00 & 44,58 & 33,73 & 1,20 & 100,00 \\
\hline $\begin{array}{l}\text { Intersection } \\
\text { Honoré Mercier/ } \\
\text { Dufferin }\end{array}$ & 14,84 & 6,04 & 2,75 & 45,60 & 27,47 & 3,30 & 100,00 \\
\hline Quartier St-Roch & 42,45 & 13,04 & 11,56 & 10,92 & 5,96 & 13,04 & 100,00 \\
\hline $\begin{array}{l}\text { Quartier Vieux- } \\
\text { Québec }\end{array}$ & 48,53 & 10,23 & 13,52 & 17,68 & 0,17 & 9,88 & 100,00 \\
\hline Québec (total) & 40,86 & 10,09 & 11,90 & 19,15 & 7,50 & 10,50 & 100,00 \\
\hline
\end{tabular}

Pour les quartiers précédemment mentionnés le tableau 3 montre qu'une forte proportion des constats émis dans le Vieux-Québec et dans St-Roch concerne les infractions liées à la consommation d'alcool et l'ébriété publique, tandis qu'une forte proportion des infractions liées aux stratégies de survie (soit le squeegee et la sollicitation) sont plus présentes sur les grandes artères, la rue Honoré Mercier, notamment à l'intersection de la rue St-Jean. On peut donc considérer que dans les carrefours routiers, on régule les stratégies de survie des personnes itinérantes, alors que dans les quartiers, on régule davantage l'ébriété publique.

Ainsi, la question du contrôle de l'espace public se pose différemment selon le type d'espace. D'un côté dans les quartiers, la sécurisation passe essentiellement par le 
contrôle de l'ébriété publique alors que sur les artères passantes, ce sont les stratégies de survie qui sont ciblées afin d'assurer la fluidité de la circulation automobile. Cette dynamique spatiale montre que la judiciarisation s'inscrit aussi dans une gestion des territoires, puisque les pratiques policières répressives s'exercent de manière ciblée et spécifique en fonction des lieux où elles se formalisent, définissant dès lors les contours d'une géographie de la répression de l'itinérance.

\section{Discussion}

L'analyse des résultats permet d'identifier des espaces autorisés et des espaces interdits aux personnes itinérantes, normativité qui s'appuie sur la régulation des conduites qu'elles adoptent. Dans la mesure où la présence directe de personnes itinérantes ne peut être réprimée, en raison notamment des protections associées aux Chartes des droits et libertés québécoises et canadiennes, les infractions reprochées vont donc s'orienter vers des conduites interdites, réprimées dans le cadre de réglementations municipales. Or, en révélant une géographie des pratiques policières répressives, notre étude permet de saisir les raisons d'être de la judiciarisation des personnes itinérantes à Québec.

En effet, l'analyse de nos données ont permis de montrer comment la judiciarisation des personnes itinérantes à Québec accompagne la revitalisation urbaine et le développement touristique des quartiers centraux de Québec, soit principalement StRoch et le Vieux-Québec. Il est toutefois important de souligner leurs limites. Tout d'abord, notre méthodologie ne nous permet pas de recenser tous les constats remis aux personnes itinérantes, mais plutôt les constats de personnes qui ont donné, au moins une fois, une adresse de refuge ou d'organisme comme adresse résidentielle au moment de l'émission du constat. En effet 3600 personnes itinérantes ont été recensées à Québec (RAIIQ, 2008) alors que notre banque de données ne compte que 284 personnes, nous sommes donc conscientes des limites de notre démarche méthodologique basée sur les adresses. Cependant, il n'en demeure pas moins qu'une personne itinérante sur 10 à Québec a reçu au moins un constat d'infraction durant la période étudiée. Par ailleurs, le lieu de l'émission du constat n'était pas fourni pour tous les constats, aussi avons-nous exclu de notre banque originale environ $31 \%$ des constats pour faire cette analyse spatiale. Malgré cela, il faut rappeler que l'objectif n'était pas d'offrir le portrait le plus précis possible du phénomène de judiciarisation des populations itinérantes (impossible, étant donné la nature même du phénomène et la situation de ces personnes en regard de leur domiciliation), mais plutôt d'en dresser les contours et les raisons d'être.

Ces premiers résultats confirment le postulat que l'on retrouve dans les écrits sur la judiciarisation de l'itinérance, à savoir que qu'elle s'exerce principalement dans les centres des villes, tantôt dans les quartiers associés depuis de nombreuses années aux territoires de l'itinérance, tantôt dans des quartiers qui, en raison de leur revitalisation, ont gagné en centralité dans les représentations (Déry et al., 2011 ; Freedman, 2009). Cependant, il est important de considérer que les centres des villes nord-américaines ont de tout temps été des quartiers centraux pour les populations marginales, si bien que la revitalisation des centres-villes vient repositionner dans ces quartiers de nouvelles populations plus favorisées, qui portent de nouvelles exigences (Freedman, 2009 ; Zeneidi-Henry, 2002). 
40 C'est dans cette perspective que notre étude témoigne des tensions entre une géographie de la revitalisation et une géographie de l'itinérance pour définir le vivreensemble dans l'espace public et le partage de l'occupation de ces espaces.

41 En effet, l'examen des quatre quartiers où la judiciarisation des populations itinérantes est la plus importante, soit St-Roch, le Vieux-Québec, St-Jean-Baptiste et St-Sauveur, ainsi que l'avenue Honoré-Mercier (entre St-Jean-Baptiste et le Vieux-Québec) montre que $92,33 \%$ des constats d'infractions sont émis dans l'arrondissement de la Cité (arrondissement fusionné avec Limoilou en 2009 pour créer l'arrondissement CitéLimoilou).

42 Cet arrondissement de la Cité semble avoir connu un renouveau démographique et économique depuis les années 1990 (Villeneuve et Trudelle, 2008), ce qui laisserait présager un processus de gentrification. Délaissé au profit des banlieues depuis les années 1950, l'arrondissement de la Cité a connu le meilleur taux de croissance démographique entre 1996 à 2001 dans tout Québec. En se basant sur les données du recensement de 2001, Villeneuve et Trudelle (2008) montrent que l'augmentation de sa population correspond en particulier à celle des jeunes adultes. Ils ont aussi observé une croissance de l'emploi local dans les nouveaux secteurs de l'économie (i.e. les services), ainsi qu'une revalorisation de la vie urbaine basée sur la proximité mais ils demeurent prudents dans leur conclusion. Pour eux, on ne peut pas conclure à une gentrification de l'arrondissement dans la mesure où ils définissent la gentrification comme l'augmentation significative du statut socioéconomique d'un quartier, mesuré par la scolarité, le statut professionnel et les revenus de ses résidents, ainsi que les valeurs résidentielles. Mais si on adopte la définition de la gentrification de Cumming dans Pivot (2008), soit un processus qui inclut la revitalisation culturelle du vivre en ville, un aspect de (re)développement économique, par le biais d'investissements privés dans les centres urbains, qu'ils soient privés, publics ou mixtes, ainsi que la hausse du coût du loyer et la réduction du parc locatif (Freedman, 2009 ; Ville de Québec, 2005), alors les résultats avancés par Villeneuve et Trudelle (2008) permettent de conclure à une gentrification de l'arrondissement de la Cité.

Pour les quartiers dans lesquels les plus forts nombres de constats sont enregistrés, soit le quartier St-Roch et le Vieux-Québec, plusieurs écrits démontrent que ces quartiers ont été au cœur d'importantes vagues de revitalisation urbaine (Ligue des droits et libertés, 2012). Le quartier St-Roch est le quartier où la gentrification semble avoir été la plus forte et la plus fulgurante (Bourgeois, 2008). Lancée par le parti "Rassemblement populaire ", elle a débuté avec le plan de relance « RevitalisAction au cœur de la capitale» (Freeman, 2009; Mercier, 2009 ; Bourgeois, 2006 ; Désormeaux, 2006). Ce plan visait la multiplicité des usages (zonage qui permet studio d'artiste et habitation, par exemple), ayant pour but de développer St-Roch comme un milieu de vie (Freeman, 2009). RevitalisAction visait un développement économique basé sur les secteurs d'activité déjà présents dans le quartier (enseignement, arts et culture), ainsi que la participation des résidents à la revitalisation de leur quartier (Désormaux, 2006). Le développement des Jardins St-Roch suivi, au début des années 2000, du démantèlement du Mail St-Roch ont marqué la revitalisation urbaine du quartier (Désormaux, 2006). Depuis, le projet de revitalisation de St-Roch a toujours cours et ne semble pas ralentir. Lors du dévoilement du plan d'action 2009-2014, le maire de la ville de Québec, Régis Labeaume, a exprimé son désir de faire du quartier St-Roch le quartier 
le "plus fou au pays " par le biais de ce plan qui fait de la promotion de la culture le principal facteur de développement (Masson, 2009). aussi documentée, mais dans une moindre mesure. Villeneuve et Trudelle (2008) soulignent que le Vieux-Québec est un quartier à usages multiples (résidentiel, touristique et commercial) et que la dynamique de revitalisation et de gentrification s'est faite à la croisée de ces usages. Bien que peu d'études aient documenté la revitalisation du quartier St-Jean-Baptiste, il n'en reste pas moins qu'une hausse observée dans les coûts du loyer laisse présager un processus de revitalisation urbaine. Quant à la rue Honoré-Mercier, elle se situe au cœur des processus de revitalisation, puisqu'elle délimite le Vieux-Québec et le quartier St-Jean-Baptiste, liant ainsi la Basse et la Haute Ville. C'est pourquoi nous pouvons donc affirmer que la judiciarisation des personnes itinérantes accompagne un processus de revitalisation et de gentrification du centre urbain de la ville de Québec.

L'analyse des types d'infraction reprochés aux personnes itinérantes indique une variation quant au lieu où les constats sont émis : proportionnellement, pour chaque lieu, plus de constats sont émis pour sollicitation et pour squegee sur la rue HonoréMercier, alors que plus de constats sont émis pour les infractions liées à l'alcool dans les quartiers St-Roch et Vieux-Québec. Les pratiques policières répressives construisent donc une géographie du contrôle en fonction des dynamiques de sécurisation qui paraissent les plus impérieuses : d'un côté la tranquillité de la circulation automobile, d'un autre côté la tranquillité des résidents, des commerçants. La judiciarisation ne tient donc pas tant au comportement adopté par la personne, qu'à sa qualification par les forces de police de " problématiques » soit par observation directe, soit en réponse à des plaintes de citoyens. En effet, comme le soulignent de Verteuil et ses collègues (2009), la pénalisation et la judiciarisation des personnes itinérantes qui découlent des conflits du «mieux vivre ensemble » ne s'ancrent pas seulement dans une lutte pour que les personnes itinérantes "disparaissent» des centres urbains. Les personnes itinérantes peuvent demeurer présentes dans les centres urbains mais dans des lieux dans lesquels leur présence n'est pas considérée comme problématique : ce sont ces lieux qu'elles peuvent investir (refuges, soupe populaire, centre de jour plutôt que des squats, parcs, etc,) qui changent par le déploiement d'outils pénaux.

Cependant comme l'affirment Parazelli et ses collègues (2013) dans une étude sur le partage de l'espace public à Montréal et à Québec, les pratiques de gestion et de contrôle des populations itinérantes se déclinent sous différentes formes qui vont de l'inclusion à l'exclusion. Ainsi, dans certains quartiers, le processus de normalisation, inscrit dans un imaginaire éco-sanitaire, renforce le rapport de la norme et contribue par la répression des populations itinérantes à les expulser de ces quartiers. Pour autant, d'autres pratiques gestionnaires inscrites, elles, dans un imaginaire démocratique cherchent à favoriser une négociation de la norme, en soutenant un cadre inclusif de cohabitation.

Cette étude, en montrant la géographie des pratiques policières répressives à l'endroit des populations itinérantes, permet néanmoins de saisir la teneur de ce rappel à la norme qui prévaut dans certains quartiers de Québec. En révélant les dynamiques sécuritaires associées à la revitalisation urbaine, la judiciarisation des personnes itinérantes permet de constater les limites participatives de la revitalisation, notamment en regard des conséquences qu'elle a sur les populations itinérantes 
(Freedman, 2009). Si les personnes itinérantes vivent le plus souvent cette répression comme une injustice, elle montre aussi comment la revitalisation exacerbe les rapports de pouvoir et de domination entre les «anciens» habitants d'un quartier revitalisé et les "nouveaux», en produisant de nouvelles légitimités dans la construction de la sécurité des lieux.

Ainsi, la judiciarisation, par ses modalités de régulation des populations itinérantes dans l'espace public, construit une géographie des pratiques policières répressives, qui répondent aux besoins et attentes de sécurisation de la population en général et des autorités pour soutenir l'attractivité de la ville.

En effet, force est de constater qu'en regard de l'augmentation de la répression, des motifs d'infraction utilisés dans les quartiers de Québec, les pratiques policières répressives apparaissent ciblées tant au plan des conduites réprimées que des espaces contrôlés dans la mesure où leur légitimité s'inscrit dans la production d'un ordre public sécurisant dans ces quartiers revitalisés. Or, notre étude montre que cette nouvelle image pacifiée des quartiers se structure davantage autour d'une expulsion par la répression des personnes itinérantes, que par une inclusion dans des logiques de cohabitation (Parazelli et al., 2013).

\section{Conclusion}

La judiciarisation des personnes itinérantes s'est cristallisée dans un espace particulier, celui des centres-villes. L'objectif de cet article était d'examiner comment la judiciarisation des personnes itinérantes se déploie dans l'espace urbain et produit une géographie des pratiques policières répressives. Dans le cas de la ville de Québec, nos résultats démontrent que la judiciarisation des personnes itinérantes est concentrée dans les quartiers en voie de revitalisation. Or, cette tension entre une géographie de la revitalisation et une géographie de l'itinérance dans les centres-villes, révélée à travers le déploiement de pratiques policières répressives ciblant les populations itinérantes, marque la construction des frontières de l'ordre établi, où la sécurité des uns légitime l'exclusion des autres. En s'intéressant aux pratiques de sécurisation des quartiers revitalisés, il devient alors possible d'aborder les zones d'ombre de la revitalisation, en caractérisant les dynamiques normatives qui prévalent pour exclure, plutôt que de ne retenir que l'éclatante réussite de la pacification urbaine.

\section{BIBLIOGRAPHIE}

Amster R., 2004. Street People and the Contested Realms of Public Space. New York, LFB Scholarly Publishing LLC.

Amster R., 2003. Patterns of exclusion: Sanitizing space, criminalizing homelessness. Social Justice, 30, 1, p. 195-221. 
Atkinson R., 2003. Domestication by Cappuccino or a Revenge on Urban Space ? Control and Empowerment in the Management of Public Spaces. Urban Studies, 40, 9, p. 1829-1843.

Bacqué M-H., Divay G., Rose D., Séguin, A-M., Sénécal G., 2003. Survol de quelques politiques de revitalisation urbaine. Montréal, INRS-Urbanisation Culture et Société, 115 p.

Bauman Z., 2000. Social issues of law and order. The British Journal of Criminology 2 40, 2, p. 205-221.

Beckett K., Herbert S., 2008. Dealing with disorder. Social control in the post-industrial city. Theorical Criminology, 12, 1, p. 5-30

Beckett K., Western B., 2001. Governing Social Marginality: Welfare, Incarceration and the Transformation of State Policy. Punishment and Society, 3, 1, p. 43-59.

Bellot C., Raffestin I., Royer M.-N., Noël V., 2005. Judiciarisation et criminalisation des populations itinérantes. Rapport de recherche au Secrétariat National des sans-abri.

Bellot C., Morselli C., 2002. Racines et enjeux de la tolérance zéro. Les politiques sociales, 1, 2, p. $4-11$.

Bernard J., 2005. Dossier : prisons : peine du pauvre, pauvre peine. Dedans/dehors, n. 47, p. 10-24.

Bernier D., Bellot C., Sylvestre M-E., 2012. La judiciarisation de l'itinérance à Québec. Rapport de recherche, Homeless HUB. http://www.homelesshub.ca/Library/La-judiciarisation-despersonnes-en-situation-d\%E2\%80\%99itinerance--a-Quebec---point-de-vue-des-acteurs-sociojudiciaires-et-analyse-du-phenomene-52808.aspx

Berti M., Sommers J., 2010.The Streets Belong to People That Pay For Them: The Spatial Regulation of Street Poverty in Vancouver, British Columbia. In D. Crocker and V. M. Johnson (eds), Poverty, Regulation and Social Justice: Readings on the Criminalization of poverty, Halifax, Fernwood.

Blomley N., 2010. The Right to Pass Freely: Circulation, Begging and the Bounded Self. Social \& Legal Studies, 19, 3, p. 331-350.

Bouchard A., 1995. L'exode des punks : le bordel à l'esplanade. Le Soleil, 17 août, p. A3.

Bourgeois F., 2008. La revitalisation du quartier Saint-Roch (ville de Québec) et ses effets sur l'expérience d'exclusion des femmes itinérantes. Mémoire de maîtrise en sociologie, Université Laval.

Castel R., 1995. La métamorphose de la question sociale: une chronique du salariat. Paris, Fayard.

Chantraine G., 2004. Par-delà les murs. Paris, Presses Universitaires de France.

Coleman R., 2004. Reclaiming the Streets. Surveillance, social control and the city. London, William Publishing.

Collins D., Blomley, 2003. Private Needs and Public Space: Politics, Poverty and Anti-Panhandling By-Laws in Canadian Cities. In New Perspectives on the Public-Private Divide, Law Commission of Canada, Vancouver, UBC Press, p. 40-67.

Déry C., Hupé P-É., Michand-Beaudry, R., 2011. Incivilités et judiciarisation : Représentation sociale dans la ville de Québec. Québec, Département de sociologie, Université Laval.

Désormeaux R., 2007. Rues St-Joseph et quartier St-Roch : la vie devant soi. Continuité, 111, p. 24-26.

DeVerteuil G., May J., Von Mahs J., 2009. Complexity not collapse: recasting the geographies of homelessness in a 'punitive' age. Progress in Human Geography, 33, 5, p. 646-666. 
Divay G., Séguin A-M., 2004. La lutte territorialisée contre la pauvreté : examen critique du modèle de revitalisation urbaine intégrée. Lien social et politiques, 52, p. 67-79.

Doherty J., Busch-Geertsema V., Karpuskiene V., Korhonen, J., O’Sullivan E., Sahlin, I., Tosi A., Petrillo A., Wygnanska J., 2008. Homelessness and exclusion: regulating public space in European Cities. Surveillance \& Society, 5, 3, p. 290-314.

Dufour R., 1998. Problématique de la Place D'Youville : Perspective d'action dans un cadre de recherche. Direction de la santé publique de Québec.

Fecteau J.-M., 2004. La liberté du pauvre sur la régulation du crime et de la pauvreté au XIXe siècle. Montréal, VLB Éditeurs.

Fleury E., 2002. Le pénitencier pour un récidiviste du « squeegee ». Le Soleil, 2 décembre, p. A1.

Freedman M., 2009. Se reconnaître dans un centre-ville en revitalisation. Expériences d'itinérants dans le quartier Saint-Roch à Québec. ACME, An international E-Journal for Critical Geographies, 8, 1, p. $100-122$.

Gueslin A., 2013. D'ailleurs et de nulle part. Mendiants, vagabonds, clochards, SDF en France depuis le Moyen Âge. Paris, Fayard.

Harcourt B., 2006. L'illusion de l'ordre. Incivilités et violences urbaines : tolérance zéro ? Paris, Descartes et Cie.

Laberge D., Roy S., 2001. Pour être, il faut être quelque part : la domiciliation comme condition d'accès à l'espace public. Sociologie et sociétés, 33, 2, p. 115-131.

Ligue des droits et libertés (Section Québec), 2012. Abus policiers et accès aux services juridiques à Québec : vécu des personnes marginalisées et point de vue des intervenant-e-s. Québec, Ligue des droits et libertés.

Mary P., 2003. Insécurité et pénalisation du social. Bruxelles, Labor.

Masson F., 2009. Saint-Roch deviendrait le quartier le plus fou au pays Québec horizon culture : projet de plan d'action 2009-2014. Québec Hebdo, le 21 janvier 2009.

Mitchell D., 2003. The Right to the City: Social Justice and the Fight for Public Space. N.Y, Guilford. Mitchell D., 1997. The annihilation of Space by Law: The Roots and Implications of AntiHomelessness Laws in the United States. Antipode, 29, 3, p. 303-337.

O'Grady B., Gaetz S., Buccieri K., 2011. Can I See Your ID? The Policing of Youth Homelessness in Toronto. Toronto, JFCY \& Homeless Hub.

Parazelli M., Bellot C., Gagné J., Gagnon E., Morin R., 2013. Les enjeux du partage de l'espace public et sa gestion à Montréal et à Québec. Perspectives comparatives et pistes d'action. Rapport de recherche remis au FQRSC.

Parazelli M., 2010. Une gestion écosanitaire de l'urbanité ? Le cas des jeunes de la rue à Montréal. In Danic I., Depeau S., David O. (dir.), Les enfants et les jeunes dans les espaces du quotidien, Rennes, Presses de l'Université de Rennes, p 205-220.

Pichon P., 2009. SDF, sans-abri, itinérant. Osons la comparaison. Louvain, Presses Universitaires de Louvain.

Pivot, 2008. A Special report on Housing Solutions for the Downtown Eastside. Vancouver, Pivot Legal Studies.

RAIIQ., 2008. L'itinérance à Québec. Rapport statistiques. 
Rousseau M., 2008. La gentrification comme politique de développement urbain? Autour des villes « perdantes ». Espaces et sociétés, 132-133, 1, p. 75-90.

Sassen S., 2002. Locating cities on global circuits. Environment \& Urbanization, 14, 1, p. 13-30.

Sibley D.,1995. Geographies of exclusion. New-York, Routledge.

Smith N., 1996. The new urban frontier: gentrification and the revanchist city. London, Routledge

Sylvestre, M-E., 2010. Disorder and public spaces in Montreal: repression (and resistance)

through law, politics, and police discretion. Urban geography, 31, 6, p. 803-824.

Vanneste C., 2001. Les chiffres de la prison. Paris, L'Harmattan.

Ville de Québec, 2005. Plan directeur. Qurtier St-Sauveur. Québec.

Villeneuve J., Trudelle A., 2008. Retour au centre à Québec : la renaissance de la Cité est-elle durable? Recherches sociographiques, 49, 1, p. 25-45.

Wacquant L., 2004. Punir les pauvres. Le nouveau gouvernement de l'Insécurité Sociale. Paris, Agone.

Wagniart J.-F., 1999. Le vagabond à la fin du XIX ${ }^{e}$ siècle. Paris, Belin.

Walby K., Lippert R., 2012. Spatial Regulation, Dispersal and the Aesthetics of the City:

Conservation Officer Policing of Homeless People in Ottawa, Canada. Antipode, 44, 3, p. 1015-1033.

Wilson J.-Q, Kelling G., 1982. Broken windows. Atlantic Monthly. 3, p. 29-38.

Zeneidi-Henry D., 2002. Les SDF et la ville. Paris, Éditions Bréal.

\section{NOTES}

1. Au Québec, la question du sans-abrisme, des populations sans-domicile fixe est définie à travers le phénomène de l'itinérance. Les personnes SDF sont alors qualifiées de personnes en situation d'itinérance (voir Pichon, 2009).

2. Cette transformation de place publique en parc n'est pas anodine puisque ce changement de statut impose des horaires d'ouvertures et de fermetures aux espaces publics. Généralement, les parcs sont fermés entre minuit et six heures du matin. Ce couvre-feu, orchestré par le changement de statut, impose aux personnes itinérantes de sortir des parcs, sous peine de recevoir une contravention

3. De nombreux bancs ont été changés dans les parcs au Québec pour éviter que les personnes ne puissent s'y allonger, la transformation de l'éclairage des parcs, la mise en place de barrières, constituent autant d'exemples de transformation du mobilier urbain, pour freiner la fréquentation des parcs par les personnes itinérantes.

4. Pour cette étude, nous avons strictement réalisé une méthodologie quantitative. Par conséquent, la pratique policière documentée est celle repérée à travers l'émission de constats d'infraction à l'endroit de populations itinérantes. D'autres recherches portant sur le même objet, utilisant des méthodologies qualitatives (entrevues auprès des personnes itinérantes, des acteurs socio-judiciaires, observations participantes) ont permis de faire état du spectre des pratiques : de la tolérance à la répression. Pour autant, ici l'article ne peut porter que sur les pratiques policières répressives.

5. En s'intéressant à la judiciarisation des populations itinérantes, à travers les pratiques de contraventionnalisation des forces policières, c'est-à-dire de l'utilisation de constat d'infraction émis en vertu de réglementations municipales et provinciales, cette étude s'inscrit dans une analyse de la mise en œuvre du droit pénal. Or, au Canada, le droit répressif se divise en deux branches: le droit criminel qui est de compétence fédérale, et le droit pénal qui est de 
compétence provinciale. Le droit pénal considéré comme un droit « mineur » dans la mesure où il n'implique pas de conséquence lourde en termes de sanction (amende généralement), s'appuie sur une procédure plus allégée, notamment par le fait que le fardeau de la preuve est renversé. Il appartient aux personnes qui font l'objet d'un constat d'infraction, de le contester dans les 30 jours, à défaut de contestation, elles sont reconnues coupables. Or, cet allégement de la preuve en matière pénale conduit le plus souvent les policiers à négliger la rédaction des motifs précis de l'accusation sur le constat d'infraction dans la mesure où peu de constats d'infraction sont contestés et que même en cas de contestation, le juge entendra la perception de la situation par le policier et la perception de la situation par la personne contrevenante. Le débat ne porte que peu sur des données objectives. Par exemple, pour la mise en accusation d'ébriété publique, il suffira que le policier puisse évoquer sa perception de l'ébriété de la personne, pour que l'infraction soit reconnue. C'est dans ce contexte, que de nombreux constats d'infraction étudiés ne comportaient pas de mention de lieu de l'infraction.

6. La définition des quartiers s'appuie donc sur une division administrative et ne suit pas d'autres découpages fonctionnels, identitaires ou historiques.

7. Il n'est pas précisé s'il s'agit d'une interdiction pour un enfant ou un adulte mais cette interdiction fait partie des interdictions présentes dans le Règlement. Par ailleurs, il faut comprendre que l'ensemble de ces réglementations municipales peuvent parfois paraitre pour le moins surprenantes mais il n'en demeure pas moins que ce sont des infractions pénales qui sont utilisées pour judiciariser des personnes. Ainsi, parmi les données complètes de l'étude, notons que nous avons retrouvé quelques infractions pour « avoir proféré des sacres ou injures dans un lieu récréatif ».

8. Il s'agit le plus souvent de jeunes qui nettoient les vitres des voitures au carrefour. Le squeegee est le terme qui désigne le balai-nettoyeur qu'ils utilisent et qui par extension est devenu le terme courant pour désigner cette pratique voire même les personnes qui le pratiquent.

\section{RÉSUMÉS}

La question de l'occupation de l'espace public par les populations SDF fait l'objet d'un contrôle policier et d'une judiciarisation de plus en plus importante dans la plupart des villes occidentales. Le déploiement de stratégies de contrôle tient en partie aux mutations des politiques urbaines, aux transformations de la police de proximité mais aussi aux enjeux que soulève la revitalisation urbaine dans le cadre de la compétition mondiale entre les villes. En cherchant toutes à promouvoir leurs richesses, leurs potentialités et leur qualité de vie, elles sont contraintes de réguler et d'invisibiliser la misère de leurs rues. C'est dans ce contexte que nous avons étudié la judiciarisation de l'itinérance dans différentes villes canadiennes. L'objectif de cet article est de montrer comment la géographie des pratiques policières répressives suit largement les stratégies de revitalisation urbaine mises en œuvre, à partir d'une analyse des contraventions émises auprès des populations itinérantes à Québec.

The occupation of public spaces by homeless people is increasingly controlled and repressed by public and private police in several Western cities. This penalization of homeless people is in part connected to changes in urban policies, transformations in community policing strategies and practices but also to socio-spatial gentrification issues.. As cities compete to be ever more prosperous, dynamic, attractive and rich, they try to make poverty invisible. Our study on the 
penalization of homeless people in various Canadian cities fits in this broader context of penalization of poverty and homelessness. The purpose of this paper is to show how the spatialization of police repressive practices are linked to the revitalization of certain neighborhoods in Quebec City by considering where tickets have been issued to homeless people.

\section{INDEX}

Mots-clés : SDF, itinérance, judiciarisation, espace public, revitalisation

Keywords : Homelessness - penalization - public spaces - gentrification

\section{AUTEURS}

\section{CATHERINE CHESNAY}

Catherine Chesnay, catherine.chesnay@uottawa.ca, est Doctorante à Université d'Ottawa. Elle a publié récemment :

- Sylvestre M.-E., Bellot C. et Chesnay C., 2012. De la justice de l'ordre à la justice de la solidarité : une analyse des discours légitimateurs de la judiciarisation de l'itinérance au Canada. Droit et société, $\mathrm{n}^{\circ} 81$, p. 299-320.

\section{CÉLINE BELLOT}

Céline Bellot, celine.bellot@umontreal.ca, est Professeure à l'École de service social, Université de Montréal.Elle a publié récemment :

- Bellot C., Bresson M., et Jetté C., 2013. Le travail social et la nouvelle gestion publique : enjeux et défis. St-Foy, PUQ.

- Bellot C., Sylvestre M.-E., St-Jacques B., 2013. Construire un problème social et pourquoi pas ? Le cas de la judiciarisation de l'itinérance. In M. Otero et $\mathrm{S}$. Roy, (eds), Qu'est-ce qu'un problème social aujourd'hui ? Repenser la non-conformité. St-Foy, PUQ, p. 207-228.

- Goyette M., Bellot C. et Sylvestre M.-E., 2014. La gestion de l'ordre public : de la confiance des citoyens à la méfiance à l'endroit des pratiques répressives. In Bernier R., Les défis québécois : conjonctures et transitions. St-Foy, PUQ, p. 198-235.

- Sylvestre M.-E., Bellot C. et Chesnay C., 2012. De la justice de l'ordre à la justice de la solidarité : une analyse des discours légitimateurs de la judiciarisation de l'itinérance au Canada. Droit et société, $\mathrm{n}^{\circ} 81$, p. 299-320.

- Sylvestre M.-E., Bernier D. et Bellot C., 2014. Red Zones Orders in Canadian Courts and the Reproduction of Socio-Economic Inequality. Onati Socio-Legal Series

\section{MARIE-ÈVE SYLVESTRE}

Marie-Ève Sylvestre, msylvest@uottawa.ca, est professeure et vice-doyenne à la recherche et aux communications à la Section de droit civil de la Faculté de droit de l'Université d'Ottawa. Elle a publié récemment :

- Bellot C., Sylvestre M.-E., St-Jacques B., 2013. Construire un problème social et pourquoi pas ? Le cas de la judiciarisation de l'itinérance. In $\mathrm{M}$. Otero et $\mathrm{S}$. Roy, (eds), Qu'est-ce qu'un problème social aujourd'hui ? Repenser la non-conformité. St-Foy, PUQ, p. 207-228.

- Goyette M., Bellot C. et Sylvestre M.-E., 2014. La gestion de l'ordre public : de la confiance des citoyens à la méfiance à l'endroit des pratiques répressives. In Bernier R., Les défis québécois : conjonctures et transitions. St-Foy, PUQ, p. 198-235. 
- Sylvestre M.-E., Bellot C. et Chesnay C., 2012. De la justice de l'ordre à la justice de la solidarité : une analyse des discours légitimateurs de la judiciarisation de l'itinérance au - Sylvestre M.-E., Bernier D. et Bellot C., 2014. Red Zones Orders in Canadian Courts and the Reproduction of SocioEconomic Inequality. Onati Socio-Legal Series

- Sylvestre M.-E., 2010. Disorder and Public Spaces in Montreal: Repression (and Resistance) through Law, Politics and Police Discretion. Urban Geography, 31 (6), p. 803-824. 